\title{
Ettore Majorana as a guide in Quirino Majorana's experiments. Original letters and documents on an experimental and theoretical collaboration
}

\author{
Giorgio Dragoni \\ University of Bologna \\ Via Irnerio 46 Bologna 40126, Italy \\ E-mail: dragoniabo.infn.it
}

\begin{abstract}
It is offered a preview of the lengthy, intense and rich exchange of scientific correspondence (currently in press) which took place in the period from 1931 to 1937 between Ettore and Quirino Majorana. This collection of letters provides insight into the specifically experimental and theoretical aspects tied to the study of changes in photoresistance in thin metal films exposed to light in suitable conditions and the possible identification of new effects of a photoelectric type. It also leads us to realise that many theoretical parts of the most mature papers by Quirino Majorana are attributable in fact to Ettore, and moreover allows us to identify Ettore as being, in a certain sense, the co-author of those publications and studies, thanks also to the constant role he played vis-à-vis his uncle Quirino as a methodologically astute critic and epistemological guide. It is pointed out, furthermore, that this correspondence allows us to recognise Ettore as the true author of the Lecture on Galvani that Quirino Majorana presented in Bologna in 1937, on the occasion of a great international Congress organised to celebrate the second centenary of the birth of Luigi Galvani. Finally, the article refutes the widespread and common belief that after 1933 Ettore no longer concerned himself with physics.
\end{abstract}

Ettore Majorana's legacy and the Physics of the XXI century

University of Catania, Italy

5-6 October, 2006 


\section{Introduction}

"I really hope to go to Naples": thus ended the intense and constructive correspondence between Ettore and Quirino Majorana. The letter, dated November, 16 1937, contains one of the rare moments testifying to a great joie de vivre in Ettore Majorana. Then a veil of obscurity fell over Ettore's life and work. But not silence. On the contrary, from this point of view there have been all too many interpretations concerning his mysterious disappearance, and "sure leads" identified by various parties.

Being one of the last letters written by Ettore, who, without doing injustice to anyone, may be considered one of the greatest, if not the greatest physicist of his time, it becomes of essential importance to acquaint scholars, and all those interested, with the contents of these letters, which are of great human, scientific and methodological value.

\subsection{The Correspondence}

But what does this correspondence consist in? It includes 34 letters and a postcard written by Ettore to his uncle Quirino and 2 letters (and an excerpt of a letter) written by Quirino in his own hand to his nephew. To this we may add, as they relate to the same research topic, 6 letters addressed to Quirino Majorana by the mathematician Dario Graffi. It should be said right away that nearly the entirety of this material is unpublished ${ }^{1}$. The brief correspondence we are talking about on this occasion is part of a much vaster archive that the Majorana family (to whom we acknowledge our thanks), above all through the initiative of Mrs. Silvia Toniolo Majorana, Quirino's daughter, has chosen to donate to the University of Bologna (for the Physics Museum ${ }^{2}$ ), where Quirino Majorana, as Director of the Institute of Physics, engaged in teaching and experimental activity for over thirty years.

The specific contents of the letters in question are almost always, barring one or two exceptions, exquisitely scientific in nature. They testify to an intense collaboration between uncle and nephew on the subject of research undertaken by Quirino, regarding the possible existence of new photoelectric phenomena and the effects of photoresistance in thin metal films when the latter are illuminated by a suitable light. The interest in these topics, and above all the manner in which they are addressed - also in view of later and current developments in the scientific research in question, as well as the careful methodological procedures contained therein - is very high.

Indeed only about ten letters were published on E. Recami, Il Caso Majorana, Arnoldo Mondatori, Milan 1987 and following editions. University

Physics Museum of the University Museum System and the Bologna Department of Physics, Bologna 


\subsection{Historical relevance of the correspondence}

Moreover, the historical value of what is contained in this close exchange of correspondence grows in importance when one considers that, according to Ettore's most intimate friends, co-members of Fermi's Group at the Institute of Physics in Via Panisperna in Rome - and whose opinion is shared by many historians - in those years (1933-1938) Ettore Majorana practically no longer concerned himself with physics. It was actually known from a letter of 1936, again addressed to his uncle Quirino, that he was still devoting himself to physics: "I have been working for some time on quantum electrodynamics." was but an isolated and, as it were, generic allusion.

In actual fact, not only was Ettore still interested in physics, but he also followed, or rather guided - as will become apparent from the reading of these letters of the collection of letters currently in press, and from the excerpts we offer in this presentation - the sophisticated, refined and difficult experiences of his uncle.

These letters thus bring to light an unexpected but humanly and scientifically rich image of the only Italian physicist who, at least up to now, succeeded in obtaining a university chair in theoretical physics by virtue of his clear fame!

Our presentation aims to pay reverent, respectful and due homage to Ettore and Quirino Majorana, on the one-hundredth anniversary of Ettore's birth.

\section{The experimental research of Quirino Majorana on metallic photoresistance}

These were experimental studies conducted by Quirino Majorana between 1925 and 1940 (circa) on phenomena of photoresistance in thin metal films and, in particular, the increase in electrical resistance that metal films undergo when exposed to light, especially if periodically interrupted. In these experiments Quirino observed that the thermal action of light - to which these effects are traditionally ascribed - does not suffice on its own to explain all the characteristics of the effects in question. Quirino Majorana identified, or believed to have identified, a new effect or effects of a photoelectric type - to be added to the classic one earlier discovered by Hertz (1886), studied and theoretically interpreted by Einstein (1905), and whose famous quantum-based interpretation was experimentally verified by Millikan (1916).

But here is what Quirino has to say: «The experimental studies on this subject, already previously reported, have now continued, being limited to the case of gold films of varying thickness, fixed or deposited on glass. The results now obtained are described, with a distinction made according to the method by which the films themselves were prepared. Then follows the presentation of a theory of the observed phenomena, considered as being due to the pure thermal effect. It is pointed out, finally, that the results of this theory do not always coincide with the experimental ones. This confirms the hypothesis that there actually exists a new effect

3 E\&QM Correspondence, Letter of E. to Q. from Rome June 11, 1936 
of light on metal films; this effect is almost always accompanied by the common thermal effect and at times remains completely concealed by it.» ${ }^{4}$

\subsection{Quirino's research}

The research in question, to which reference is made here by citing the more general and almost conclusive paper written by Quirino in 1938, has a long story behind it, which is thus described by the Author:

«In the lengthy experimental period I have dedicated to this research, I have noticed that the observed effects depend on numerous circumstances. They are: the nature and thickness of the metal constituting the film; the method of fixing it on an insulating substrate (glass, quartz, ebonite, etc.), using an adhesive substance; or generating it on the substrate itself by chemical, thermal, mechanical or cathodic precipitation; exposing the other surface of the film, fixed as just described, to water or a gas made to flow over it at a higher or lower speed; using the film without any substrate (just supporting it at either end) immersed in a gas (air or hydrogen), or in a vacuum; and, finally, changing the nature and intensity of the source of radiation and the frequency of interruption of the latter.» ${ }^{5}$
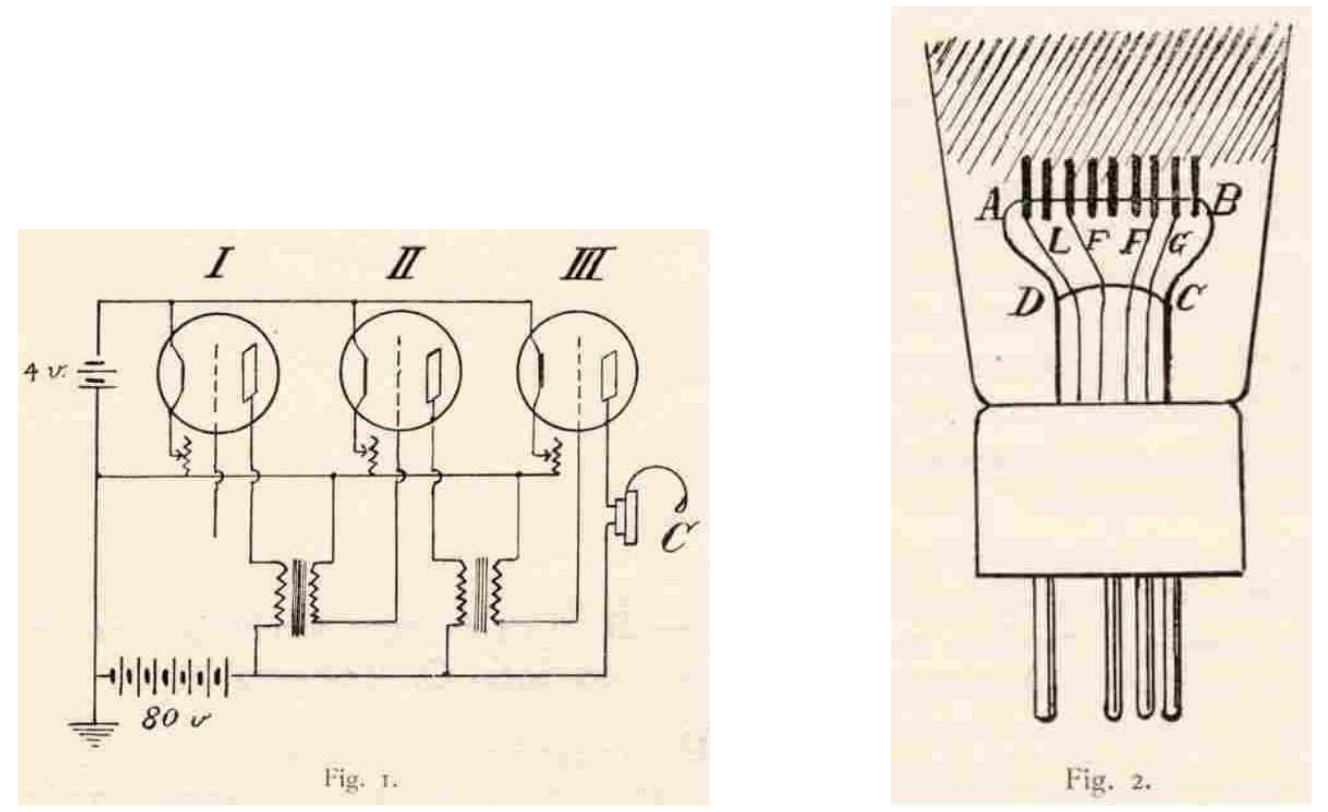

The drawings in Fig. 1, Fig. 2 and Fig. 3 respectively represent the circuit employed, the the photoelettric cell (audion) ${ }^{6}$ and the principal and most refined experimental device devised by Quirino Majorana in the course of his experiments is illustrated in the following figure:

\footnotetext{
4 Q. Majorana, Ulteriori ricerche sull'azione della luce su sottili lamine metalliche, in "Il Nuovo Cimento", 15 (1938) 573-593; 573

Ibidem, 573

Q. Majorana, Su di un fenomeno fotoelettrico constatabile con gli audion, "Rendiconti Accademia dei Lincei” 7 (1928) 801-806; 802-803
} 


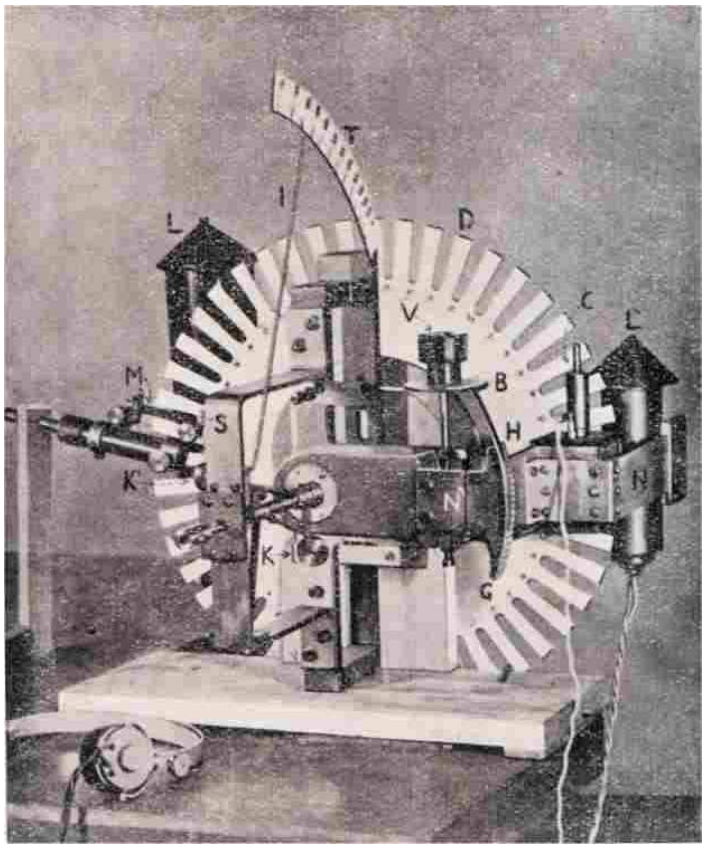

Fig. 3

Fig. 3 represents, specifically, the main apparatus which is thus described by Quirino Majorana. $« D$ is rotating disc of elektron with a diameter of $50 \mathrm{~cm}$, whose periphery is provided with 48 radial indentations. The width of the indentations is equal to that of the solid parts. With this, the light from a lamp $L$ (which can be an incandescent or mercury-quartz lamp with power adjustable to up to around 900 and 300 watts, respectively), periodically arrives on a common glass plate $M$ bearing the metal film (gold in these experiments), prepared according to one of the methods mentioned. The rotation speed of the disc is set in these trials so as to generate, usually, a frequency $n$ of interruption of the light ray equal to 600 or 1200 periods per second. By means of a centrifugal force device $K K^{\prime}$, supported by a bracket $S$, and a pointer $I$ sliding over the graduated scale $T$, it is possible in any case to control the value and constancy of this frequency. $C$ is a potassium photoelectric cell in a vacuum; $L^{\prime}$ is a subsidiary adjustableintensity incandescent lamp which illuminates $C$ through the indented edge of the rotating disc $D$. The cell $\mathrm{C}$ and lamp $L^{\prime}$ are borne by an arm $N N^{\prime}$ keyed to the rotating shaft of the disc, which can be shifted angularly around this by means of a micrometric screw $V$, which bears a pointer sliding over a graduated horizontal ring $B$. The relative angular position between the two systems lamp $L$ - plate $M$ and lamp $L^{\prime}$ - cell $C$ may be identified by taking readings on the graduated sector $H G$ (having its centre on the disc shaft) and ring $B$. Special protections, not illustrated in the figure, prevent the air currents generated by the rotating disc from acting upon $M$. The apparatus is connected with the other accessories indicated in the compensator layout published previously. It thus achieves the formation, amplification and compensation of the pulsing current which characterises the effect in question.»

$7 \quad$ Q. Majorana, Ulteriori ricerche..., 576 


\subsection{Experimental results}

The above-described apparatus makes it possible to determine «the value of $\Delta \mathrm{r} / \mathrm{r}$ (maximum change in the electrical resistance of the film) and the phase shift $\varphi$ between the pulsation of the light and that of the electrical resistance. The magnitude $\Delta \mathrm{r} / \mathrm{r}$ is equal, as was seen, to the ratio between the peaks of the two electric currents (after compensation) which circulate in the photoelectric cell and in the metal film. The value of this magnitude gives the measure of the effect. And as I said earlier, it is to be admitted that said effect must, if anything, be of a mixed nature: thermal and photoelectric of a new nature.» ${ }^{8}$

The existence of a possible new photoelectric type effect is confirmed, or at least evidence thereof is given, shortly thereafter the article we are citing.

«In the case of glued films, these latter facts can naturally find no explanation in pure thermal theory. They give, once again, clear evidence of the existence of a photoelectric fact of a new nature.» ${ }^{9}$
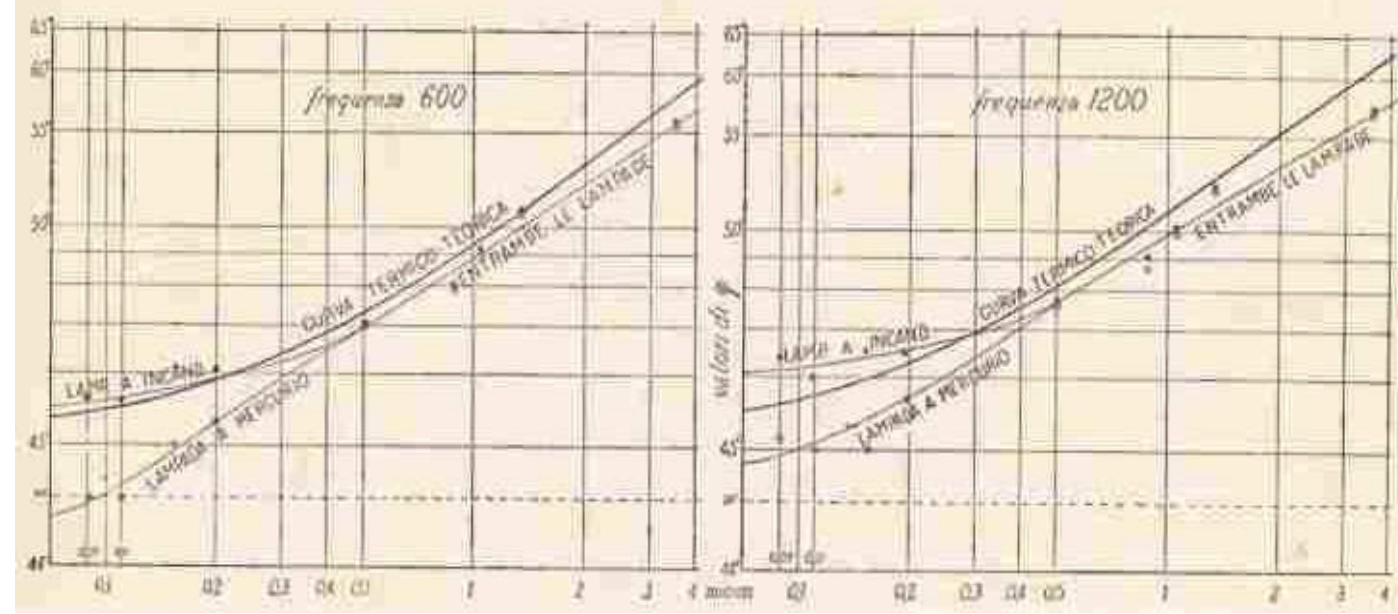

Fig. 4

The lengthy experimental work of Quirino may be condensed in two summary graphs (see Fig. 4), which are thus commented by Quirino, with a certain degree of satisfaction:

«The experimental curves diverge considerably from the theoretical-thermal ones. For small thicknesses and for each frequency they lie intermediate between the experimental ones of the two lamps. For large thicknesses they lie decidedly above, deviating by as much as about $5^{\circ}$.

These results confirm the influence of the nature of the radiation on the size of the effect. Further considerations will be made after an illustration has been given of the theoretical study of the pure thermal hypothesis, which here as well does not appear sufficient to explain all of the modes of the facts observed.» ${ }^{10}$

The fundamental point of all this work is thus to assess whether the effects identified, or believed to have been identified, are, or are not, of a typically thermal origin. At this point 
Quirino develops a detailed mathematical argumentation with the aim of identifying the exact order of magnitude of the thermal effect in his experiments and of determining, accordingly, whether the origin of the recognised effects is other than thermal.

\section{The theory of the phenomena studied}

Here's how Quirino Majorana presents the mathematical calculations that will enable him to determine the entity of the effect due to thermal variations in the metal films.

Quirino intends to study «metallic photoresistance», i.e. the increase in electrical resistance that a metal film undergoes when exposed to light. Quirino Majorana realises that, using the devices described earlier, such an effect will be more easily observable if the light is periodically interrupted.

His research has led him to the conclusion that: «the thermal action of light does not suffice on its own to explain all of the characteristics of the effect in question. It may thus be interesting to establish, based on the classic theory of heat propagation, the size of the effect predictable by such means, in order to see, afterwards, whether and how such a prediction deviates from the actually observed facts. I shall thus proceed to an illustration thereof.» ${ }^{11}$

\subsection{Theoretical development}

A very reasonable hypothesis is advanced concerning the interpretative characteristics of the phenomenon studied.

«Let us now suppose, first of all, that light rays of any wavelength fall on the film in a continuous manner. They will be partially absorbed. Let us say that this will occur to the degree of $W$ calories per $\mathrm{cm}^{3}$ of film per second. If, by contrast, said rays are delivered periodically, by means of a disc with radial indentations, we can admit that the interruption will occur according to a sinusoidal law and with an angular frequency $\omega$. This result may be achieved with sufficient approximation by suitably configuring the indentations in the disc and the profile of the metal film.

With said contrivance, the energy $w$ which is transferred to the film in each unit of time will become variable rather than constant and can be indicated with the expression:

$$
w=\frac{W}{2}(1+\cos \omega t)
$$

Under the action of this thermal energy, the temperature of the film progressively rises, in relation to the ambient temperature, until reaching a steady state, as a consequence of external heat dissipation. It may thus be said that the energy absorbed by the film in the unit of time is expressed by:

$$
q=A \cos \omega t
$$

where $A=W / 2$. This relationship establishes the pulsation of thermal energy possessed by the film. Therefore, such energy increases during one half-period and decreases during the

\footnotetext{
11 Ibidem, 584
} 
other; this is so since the absorption and of said energy and its dissipation to the outside occur continuously in a periodic mode and with opposing phases.

In such conditions, the film temperature pulses with an amplitude $T_{m}$ around a certain temperature, somewhat higher than the external ambient temperature. The phase of this periodic phenomenon is delayed, in relation to that of the light pulsation, by an angle $\varphi$. For the purposes of this discussion, it is of interest to determine only $\mathrm{T}_{\mathrm{m}}$ and $\varphi$.

Now let $\rho$ be the density, $c$ the specific heat of the metal and $\delta, e$, the analogous magnitudes for the glass. Let us say $C=c \rho \varepsilon$, the thermal capacity of the film per $\mathrm{cm}^{2} ; E=e \delta$, the thermal capacity of the glass per $\mathrm{cm}^{3}$. Let $k$, moreover, be the thermal conductivity of the glass.

The variable part of the film temperature may be indicated as

$$
T=M \cos \omega t+N \operatorname{sen} \omega t
$$

$M, N$ being unknown constants.

The anterior surface of the glass, which is in more or less perfect contact with the film, likewise has a variable temperature, $\mathrm{T}_{0}{ }^{\prime}$, which will be quite different due to the poor thermal conductivity of the adhesive and the considerable propagation of heat in glass. We can say:

$$
T_{0}^{\prime}=P \cos \omega t+Q \operatorname{sen} \omega t
$$

$\mathrm{P}, \mathrm{Q}$ being two other unknown constants. Generally speaking:

$$
\frac{N}{M} \neq \frac{Q}{P}
$$

Since the phases of the thermal pulsations of the metal and of the glass surface are different, the phase lag $\varphi$ we are looking for corresponds to:

$$
\operatorname{tang} \varphi=\frac{N}{M}
$$

the maximum amplitude of the thermal pulsation of the film is given by:

$$
T_{m}=\sqrt{M^{2}+N^{2}}
$$

It is now a question of finding four equations enabling us to determine $\mathrm{M}, \mathrm{N}, \mathrm{P}, \mathrm{Q}$. Let it be observed, first of all, that part of the heat absorbed by the film is used to increase the temperature thereof and part is transferred toward the air and the glass. It follows that:

$$
A \cos \omega t=C \frac{d T}{d t}+h T+\eta\left(T-T_{0}{ }^{\prime}\right)
$$

From this, given (2) and (3), and equating the coefficients of $\cos \omega t$ and $\sin \omega t$, it is possible to deduce two of the four equations being sought:

$$
\begin{aligned}
& A=C \omega N+(h+\eta) M-\eta P, \\
& 0=-C \omega M+(h+\eta) N-\gamma_{i} Q .
\end{aligned}
$$

To obtain the other two, we must examine the way in which heat is propagated inside the glass. The latter's surface temperature $\mathrm{T}_{0}{ }^{\prime}$ being known, the temperature at a depth $x$ may be obtained by means of a well-known procedure and is expressed by: 
where:

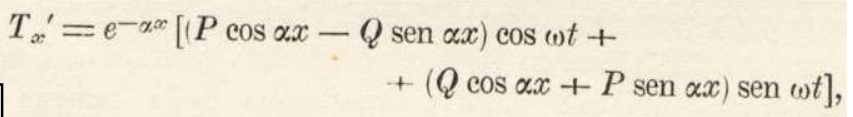

$$
\alpha=\sqrt{\frac{\overline{E \omega}}{2 k}} .
$$

The quantity of heat transmitted by the film to the glass surface must now be equated with that which penetrates from this surface toward the inside. It follows that:

$$
\eta_{i}\left(T-T_{0}^{\prime}\right)=-k\left(\frac{\partial T^{\prime}}{\partial x}\right)_{x=0}
$$

By replacing the values with those expressed in (2), (3) and (7), equating as usual the coefficients of $\cos \omega t$ and $\sin \omega t$ and assuming:

$$
p=\sqrt{\frac{E k(t)}{2}}
$$

we obtain the other two equations sought:

$$
\begin{aligned}
& \gamma_{1}(M-P)=p(P+Q), \\
& \eta(N-Q)=q(Q-P) .
\end{aligned}
$$

The four equations (I), (II), (III), (IV) must enable us to determine $M, N, P, Q$. Then to obtain $T$ and $\varphi$, it will suffice to use (5) and (4).

The written equations are thus satisfied by the following expressions:

$$
\begin{gathered}
T_{m}=\frac{A}{\sqrt{\left[C\left(\omega+p \frac{\eta^{2}}{(p+\eta)^{2}+p^{2}}\right]^{2}+\left[h+p \frac{2 p \eta+\eta^{2}}{(p+\eta)^{2}+p^{2}}\right]^{2}\right.}}= \\
=\frac{A \cos \varphi}{h+p \frac{2 p \eta+\eta^{2}}{(p+\eta)^{2}+p^{2}}}, \\
\operatorname{tang} \varphi=\frac{C\left(\omega+p \frac{\eta^{2}}{(p+\eta)^{2}+p^{2}}\right.}{h+p \frac{2 p \eta+\eta^{2}}{(p+\eta)^{2}+p^{2}}} .
\end{gathered}
$$

\subsection{Experimental and theoretical comparison}

Thus concludes the main part of the mathematical discussion. Some simplifications will be illustrated below.

In his 1938 article for "Nuovo Cimento" Majorana continues:

«To clarify the meaning of the previous formulas, one may construct a graphic representation, varying some of the parameters contained therein. It is more interesting and simpler to do this for (9); this formula gives the value of $\varphi$, which, as may be seen therefrom,

$12 \quad$ Ibidem, 584-587 
and as is largely confirmed by experience, is independent of the quantity of heat $A$ absorbed by the film, and thus also of the intensity of the rays that fall upon it.

I refer to the experimental case described previously: gold films fixed on glass. The following values may be chosen for the various parameters indicated earlier: $\rho=19.2 ; \delta=2.51$; $c=0.030 ; e=0.21 ; k=0.0017 ; h=0.00018$. Among these, the latter two (internal heat conductivity of the glass, and external heat conductivity of the gold immersed in air) posed a greater degree of uncertainty. Therefore, using the direct method, I determined $k$ for the special glass used; $h$, being very small, has little importance and I drew its value from current manuals. Two frequencies of light ray interruption were chosen: 600 and 1200; from this we may derive the two values of $\omega$. In (9), $\varphi$ and $\eta$ thus remain variable. Experimentally, as was said, no prediction can be made as to the value of $\eta$. I assume here that it may vary between the extended limits: 50 and 0.005 p. calorie per $\mathrm{cm}^{2}$ of film, per second, and per degree of thermal difference between metal and the anterior surface of the glass. Thus the graphs of figure 5 are constructed, point by point, for the aforesaid frequencies and for two different thicknesses of gold film: 0.08 and 0.7 microns. In calculating the points of these curves, we ignored the value of $h$. This is perfectly legitimate within the limits of graphic approximation.

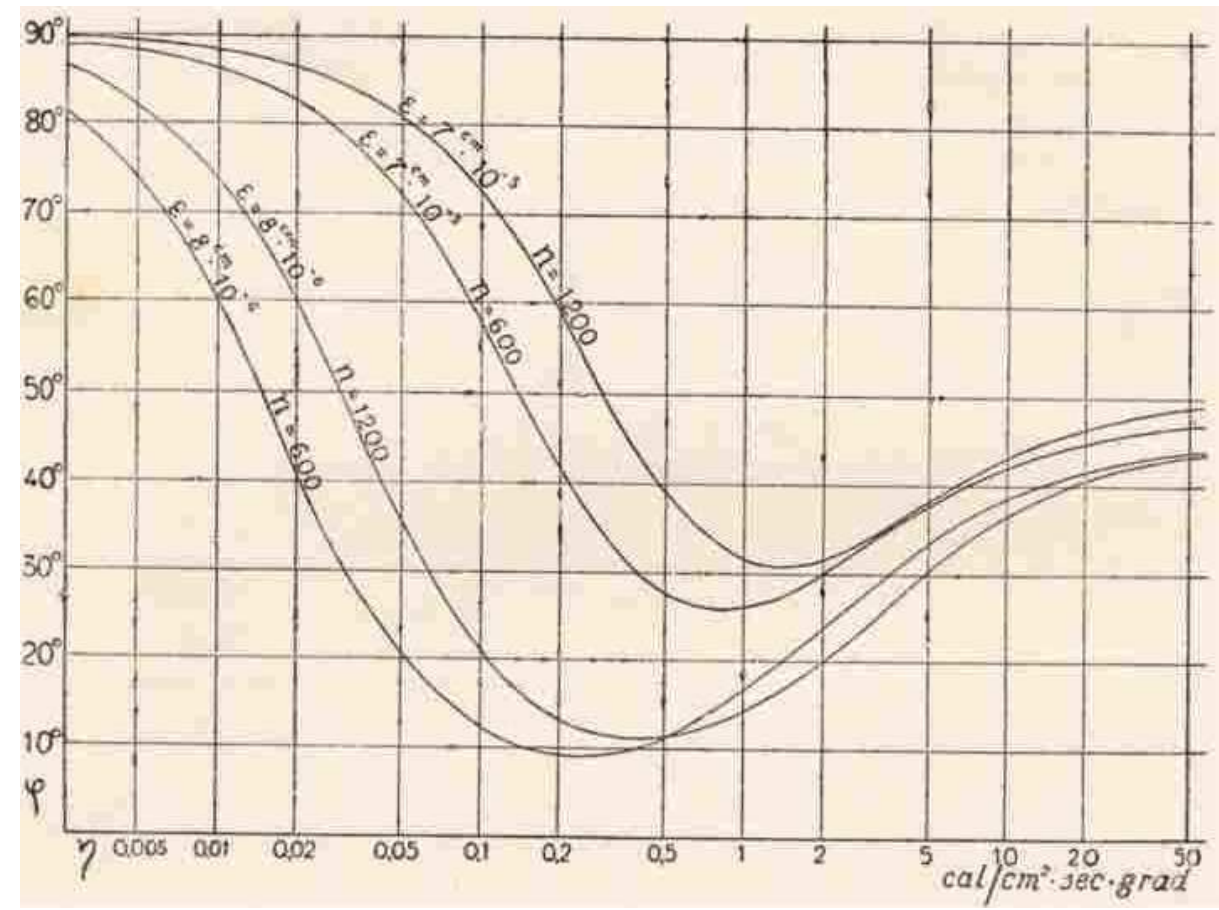

Fig. 5

The curves are based on the values of $\eta$ expressed on a logarithmic scale, to better highlight what occurs for small values; and on the arc degrees represented on a linear scale, from $0^{\circ}$ to $90^{\circ}$. They show a minimum, which, for $0.08 \mu$ films, is around $10^{\circ}$. They are asymptotic, due to the logarithmic representation, to a curve parallel to the abscissas ( $x$ coordinates) of ordinate $90^{\circ}$, for $\eta$ tending to zero (or infinite insulation). On the side of large values of $\eta$ they are still asymptotic (and they would always be so even for a linear 
representation of $\eta$ ) to an ordinate proximate to $45^{\circ}$, but slightly higher than that value, for $\eta$ tending to infinity (perfect conductivity, as for cathodic films).

Assuming permanently $\eta=\infty$, the formulas (8) and (9) are simplified, so much so that the value of $h$ becomes completely negligible. In such a case, the thermal pulsations of the film are channelled almost exclusively into the glass. Said formulas become:

$$
\begin{gathered}
T_{m}=\frac{A}{\sqrt{(C \omega+p)^{2}+p^{2}}}=\frac{A \cos \varphi}{p}, \\
\operatorname{tang} \varphi=1+\frac{C \omega}{p} .
\end{gathered}
$$

Two curves corresponding to the theoretical computation of $\varphi$, made using the formula (11), have already been plotted in figure 4 of the paragraph above, and thus compared with the experimental results.» ${ }^{13}$

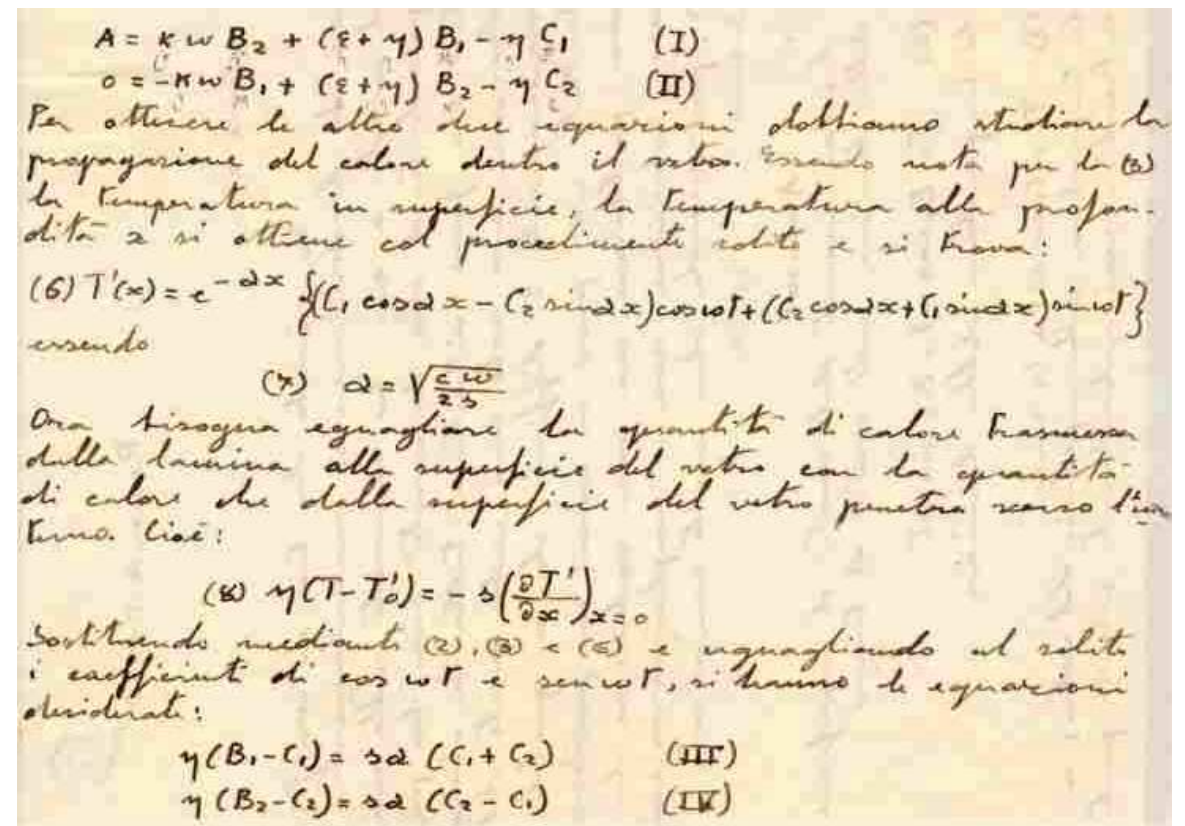

Fig. 6 - Ettore Majorana's letter addressed to Quirino (May 14, 1935). Please, note the equivalence between this writing and the one above printed.

It may perhaps surprise some that in this brief presentation such ample space has been dedicated to this theory. The reason lies in the fact that the theory - as may be inferred from the correspondence we are referring to - was written in full by Ettore Majorana. In consideration of the exiguous quantity of published writings by Ettore, it is hardly irrelevant to point out this aspect. Both for general reasons - his profound interest in physics in the years documented by the letters - and because of the light it sheds on Ettore's character.

$13 \quad$ Ibidem, 587-589 


\section{The role of Ettore}

Besides what was already known, namely that Ettore helped his uncle to confirm or formulate some calculations, in actual fact the correspondence between the two demonstrates a much more profound and complex form of collaboration. The young Ettore's role vis-à-vis his well-known uncle Quirino is in fact surprising, given the considerable age difference; that is, Ettore acted as a guide, a referee, a scientific judge - albeit an affectionate, attentive one - of his uncle's work. This initial impression becomes increasingly definite and precise with the passing of time and thus the pages of the letters exchanged between the two scientists and their respective roles are better defined and clarified. At the start Ettore acted almost exclusively as a go-between between Quirino Majorana and Enrico Fermi. Quirino appeared in fact to be anxious to know what opinion the young, brilliant Fermi held about his research. "Dear uncle, only tonight I have been able to see Fermi, who had been off for some days"(to Quirino April 17, 1931). And again, more than a year after: "If you wold like to have more authoritative comments (on the subject), I will ask Fermi, whom I hope to see next weekend." (to Quirino July 12, 1932). And the week after: "Beside the thermal hypothesis Fermi suggests two more ones, whose worth can be judged only by the experimentalist..." (to Quirino July 17, 1932). Then the role of Ettore becomes defined as that of a theoretical physicist, who develops calculations and works out formulas for his uncle: "However, the amplitude and phase can be obtained almost simultaneously with both methods, and I would have used the old calculation if I had saved it" (to Quirino September 7, 1936).

\subsection{Experimantal suggestions}

Scholars who have already had an opportunity to see these letters very often consider Ettore's role to have been limited to this behaviour, already in itself important and significant. That is, he is seen basically as a simple and occasional supervisor, perhaps in part disinterested, of his uncle's experimental research. A more complete analysis of the correspondence instead reveals an active, interested, increasingly involved participation of Ettore in his uncle's research. "You don't describe me the device that you have used..." (to Quirino February 20, 1935). Or, like when, as an example, he suggests new types of obtainable measures through variation of the luminous intensity or the frequency of the employed light: "Intensity measures could facilitate the interpretation. Perhaps the easier mean is to vary the frequency." (to Quirino May 4, 1935). Or when he raises doubts: "The fact you referred to, that part of the energy is absorbed directly by the glass rather than by the films, certainly also warrants careful examination, but I lack too many data to be able to express an opinion as to its consequences" (to Quirino May 9, 1935). And furthermore: "As regards the experiments momentarily interrupted, my impression continues to be that discussing the only results already obtained cannot lead to any conclusion." and: "It is not indispensable that on p. $8^{14}$ you ascribe me credit for an elementary calculation that you can do and redo or have checked by whoever you judge

14 The reference is probably related to "La Ricerca Scientifica", National Research Council, Azione della luce su sottili lamine metalliche, 1 (1935) 
best if you do not wish to take responsibility for it." (to Quirino August 11, 1935). Or: "The curves you sent me do not actually correspond to the formula. It certainly will not be difficult for you to discover the source of the error"(to Quirino January 23, 1937). Or yet again: "The possibility of an immediate comparison of the two curves being thus ruled out, it does not seem that any definitive conclusions can yet be drawn" (to Quirino September 17, 1936). In reality, Ettore's role in the research increases systematically over time, as when he presses his uncle with new requests, asking about further and more specific experimental results. "But as it is now too late to take advantage, I must confine myself, given the complexity of the facts observed, to venturing some rather vague suppositions and above all.... to asking you some more questions." (to Quirino July 25, 1935), or: "Naturally, in order to definitively overcome such objections it would suffice to demonstrate the stability of the results obtained with the varying of certain experimental conditions" (to Quirino August 29, 1936). And, addressing the heart of the problem: "The main question is whether the technique of the experiment, after the considerable and effective improvements you have made since the first attempts, has already reached the necessary perfection, such as to achieve the rigorous isolation of causes which is its intent. But perhaps there is no call to think so far in advance about everything you will want to do upon your return to Bologna" (to Quirino August 2, 1935).

\subsection{Methodological indications}

Another general function systematically performed by Ettore throughout their exchange, besides the one illustrated above, was to exert a cautious, respectful criticism of his uncle's enthusiasms. Like when he expressly invites him to suspend judgment: "It is possible that the observed phase lags indicate the existence of a new phenomenon, but perhaps it would not be superfluous to exercise some caution in ruling out its being a simple thermal effect;" (to Quirino May 4, 1935) or when he encourages him, implying that his uncle has adopted methodological caution by his own initiative: "There is not much to say concerning their interpretation, since you deliberately refrain from advancing hypotheses" (to Quirino August 19, 1936). Or when he pays deference to the typical role, experimental, of his uncle in research: "I have nothing else to add as a "theoretician", other than the hope that where theory is lacking, help may come from experience" (to Quirino February 20, 1935). Or else, as he seeks to placate and mitigate the annoyance expressed in the reply of his uncle, who felt he was not being believed in his experimental convictions, with a display of diplomacy and of methodological astuteness that still has resonance today: "I do not well understand why, however, there being two inverisimilitudes in conflict, one should definitively side with one of them when it is not only easy, but extremely easy, to solve the dilemma with further research. I do not think you will wish simply to reply to me that a definitely ascertained fact cannot be denied by theories and above all by theoretical assumptions. It is not, in reality, a matter of immediate, tangible evidence but rather of facts recognised with the aid of hypotheses, obvious ones to be sure, on the experimental conditions, but to which we may not, without making an excessively subjective judgment, attribute greater verisimilitude than to such theoretical assumptions. For these reasons it appears to be good method to examine other possibilities which in other 
circumstances would be rightfully ignored because a priori improbable. I thus do not find the accusation of a lack of serenity to be justified; on the contrary I have made a point of not attributing excessive, and certainly not decisive, importance to the objections I have been obliged to raise. Naturally, if you allude to this speaking about the practical purposes of life, no one can expect you to continue experiments that may constantly pose new problems and if you choose to leave this task to others I shall certainly not be the one to criticise. But it was my duty to point out to you precisely what, in my modest opinion, are the open problems at the present time. I would be truly grieved if there remained even the slightest shadow of misunderstanding. Affectionately yours, Ettore" (to Quirino September 5, 1935). Or when he reassures him about the importance of his lengthy and arduous research: "Thank you for the letter regarding engineer Baldini, which we immediately delivered to the person concerned, and for the news you give me about the new phenomena you have observed in the course of your experiments. Whatever may be their explanation, given their impressiveness, they are likely to be of considerable applicative interest." (to Quirino March 9, 1935). Or else as he mentions the possibility of conducting further, decisive verifications: "Naturally further tests (with an approximate value of 'experimentum crucis') are always possible. I shall mention two: ..." (to Quirino May 16, 1935). In the above-quoted passage Ettore Majorana demonstrates a methodological foresight that frankly surprises us. He knows very well, after all, that no experimentum crucis exists (indeed he says: "with an approximate value of 'experimentum crucis'...") but he leaves his uncle room for hope, emphasising his specific competences and role as an experimenter, fundamental in the eternal comparison between theory and experiment: "Therefore, the theoretical assumption against the result you obtained may fall in the face of experiments that give quantitatively definite and reproducible results." (to Quirino February 20, 1935). To summarize, Ettore takes upon himself the task to single out causes that may perturb the phenomenon, secondary effects, sources of error, the main physical quantities and the parameters involved, determination of their respective orders of magnitude, narrowing the research field to better isolate the phenomenon at hand ... But he also suggests techniques capable of minimizing certain effects and maximizing others, in order to depurate the main phenomenon of all possible perturbing causes. A further task that Ettore takes upon himself is that of checking - logically and methodologically - the explicit (or implicit) hypotheses made by his uncle while comparing the general physical principles with the method employed in collecting and representing graphically his data. To achieve this aim Ettore availed certainly himself of knowledges and competences he had acquired by following undergraduate courses in engineering to near fulfillment of a bachelor degree before graduating in physics (Ettore's nephew Fabio Majorana and I do quite agree on this point).

\section{Concluding remarks}

In the case we have analysed - unlike those concerning other areas and other papers published by Ettore Majorana and investigated by other colleagues, on this occasion where we have reflected upon this extraordinary figure from various perspectives - Ettore stands out not 
so much for the extraordinary quality of his calculations or the inventiveness and brilliance of his predictions and theoretical formulations, but rather for the noteworthy and truly surprising ability he showed in the supervision and methodological verification of the experimental procedures carried out by his uncle. Indubitably, since those years the sector connected to photoelectric phenomena has been extended, broadened and developed considerably

However, rather than dwelling upon this point, we wish to draw readers' attention to the importance of the aspects of general historiographic interest relating to Ettore's behaviour as a human being, to the small historiographic discoveries that have been made, and to considerations of method that may be documented through this correspondence.

\subsection{Human aspects}

With regard to the first aspect, as we read through the letters we witness Ettore's progressive withdrawal into himself. At the time he began corresponding with his uncle and in the first letters dated 1931-33 he repeatedly mentions Fermi's name, almost as if to provide an absolute guarantee of scientificity and, at the same time, manifestly, to underscore their friendship, familiar acquaintance and the esteem he enjoyed "...I have to meet him next weekend..." (to Quirino July 12, 1932). After these initial mentions, Fermi's name disappears for good from his dialogue with his uncle. From the end of 1933, Ettore proceeds to isolate himself from the Group of Via Panisperna; he almost never goes to the Institute any more "...send the letters to me at home..." (to Quirino September 6, 1933). We witness a progressive estrangement of Ettore from his friends. As we read further into the correspondence we may perceive another worrying sign - knowing how things later went - Ettore refuses to accept credit for a calculation his uncle would like to attribute to him "It is not indispensable that on p.8 you ascribe me credit for an elementary calculation that you can do and redo or have checked by whoever you judge best if you do not wish to take responsibility for it." (to Quirino August 11, 1935). Still later Ettore refuses to take credit, unless as co-author - something that would have been only right given his contribution to those studies - for the formulation of the thermal theory of the phenomenon investigated. "There's no reason to give citations"(to Quirino August 23, 1936). His uncle is worried, but not as much as the situation perhaps warranted. He thinks such behaviour is due to reasons of modesty on Ettore's part, or the simplicity of the calculations performed by his nephew, to whom he attributes extraordinary abilities: "I wanted to ask you something else. In the course of this work of mine, I often turned to you for collaboration. Already last year, you did not want me to cite your name in this connection. On the other hand, I think that in a complete account of the observed facts it would not be inappropriate to mention some of the developments that you favoured to me. With regards to this point, I ask you: Do you think this would be considered inappropriate by the readers? Do you insist in denying me the pleasure to mention your name? And, in this case, do you do it for modesty or because these matters are too simple for you? But, in this case, do you believe that your uncle could wrap himself in your doctrine without doing something beneath his dignity (subsequently corrected in reproachable)" (to Ettore August 22, 1936). He fails to realise, maybe because he loves his nephew too much, that Ettore is in the midst of a further 
involutional process, becoming estranged even from the persons closest to him and society in general. An estrangement which, as we know, will lead to his decision to disappear.

\subsection{Historiografical results}

As regards the second aspect, we may note that through this correspondence Ettore leaves us, as in all the other fields he concerned himself with, precious cultural gifts that we may include among the results of our research. We were able to identify an entire chapter within Quirino's article of 1938 which was in reality completely written and formulated by Ettore: the thermal theory of the phenomena investigated by Quirino. Another significant finding of our research - one we have yet not talked about - relates to the identification of an entire lecture traditionally attributed to Quirino, and objectively bearing his signature, but which in actual fact was completely written by Ettore: Quirino's conference on Luigi Galvani, given on the occasion of the second centenary (1937) of the birth of Galvani. As it is testified by the following quotation: "Dalla Noce ${ }^{15}$ had informed me about the congress on Galvani. I would like to participate, but shall very likely be obliged to renounce to avoid a much too long trip from Sicily. I have improvised an opening speech according to what seemed to me to be the lines you had in mind. You will probably think that I kept too much to generalities, but it didn't look easy to me to tell much more without exceeding the limits you had indicated. In any case, if you don't like it, send it back to me with your comments. I shall be in Rome within a week, and a few days later, I guess, in Sicily. The opening and closing parts, in words suitable to the occasion, are not an example of eloquence, but I added them for the possible links with the rest. (to Quirino September 1, 1937). Evidently Quirino, that had been submitted to a surgical operation a little time before, had asked for the aid of the nephew.

15 Giulio Cesare Dalla Noce, at that time lecturer for theoretical physics and secretary of the Committee for the Galvanian celebrations 


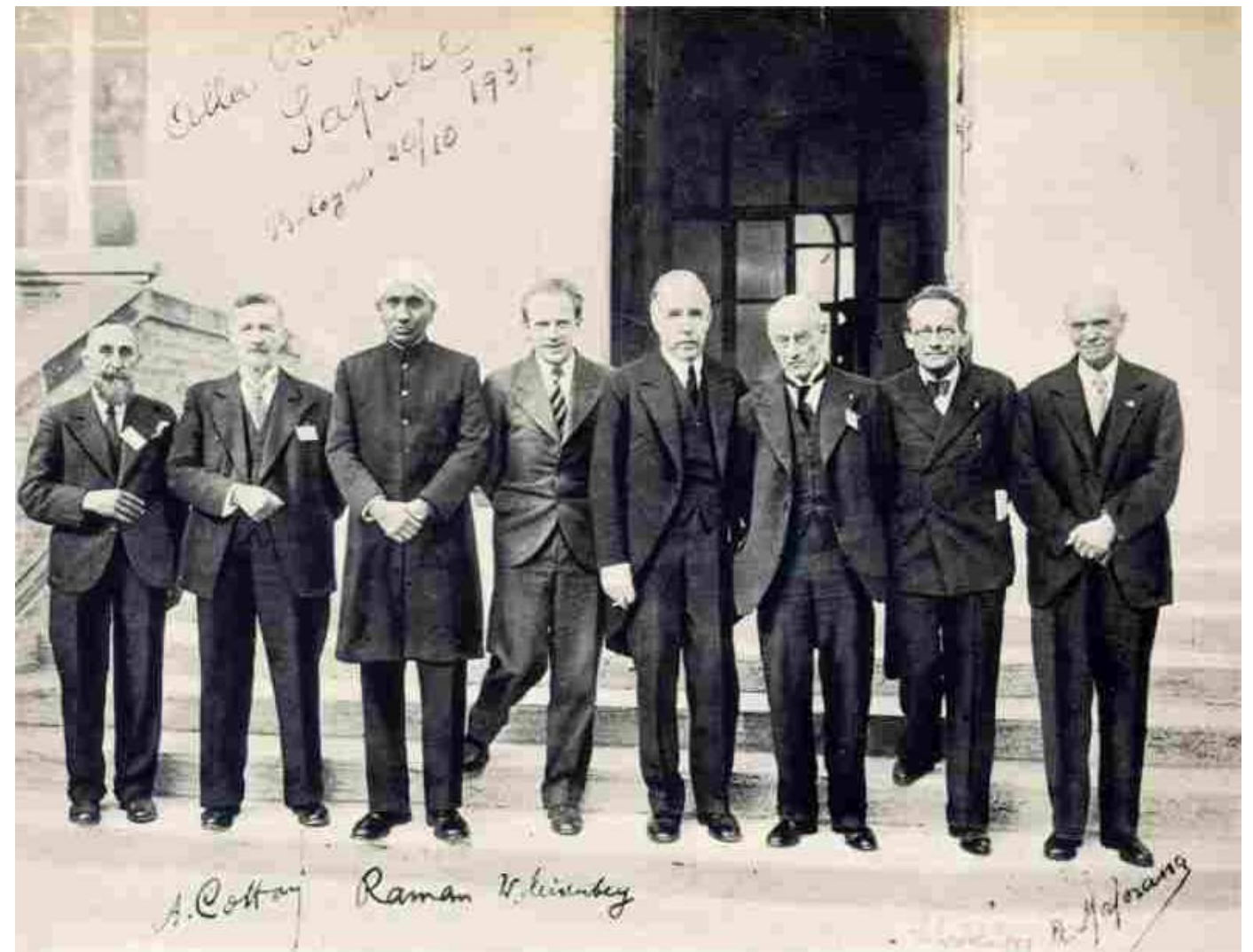

Fig. 7 - Scientists and Nobel Prize Winners participating to the galvanian celebrations in Bologna (1937). From the left: A. Turpain, A. Cotton, C.V. Raman (Nobel Prize), W. Heisenberg (Nobel Prize), Niels Bohr (Nobel Prize), O.W. Richardson (Nobel Prize), E. Schrödinger (Nobel Prize), Q. Majorana. Attending the conferences other Nobel Prizes were present: L. de Broglie, E. D. Adrian, K.M.G. Siegbahn, P.J.W. Debye, F.W. Aston and W.R. Hess. Some other participants obtained the Nobel Awards in the following decades. Full of meaning the absence of A. Einstein, perhaps due to political reasons. (Courtesy by "Sapere", October 31, 1937, p. 266)

A conference of extraordinary importance because it was held before Italy's monarchs in 1937 and above all because it was attended by the leading experimental and theoretical physicists of that time. It was a text abounding in notes of great historical and epistemological value. 


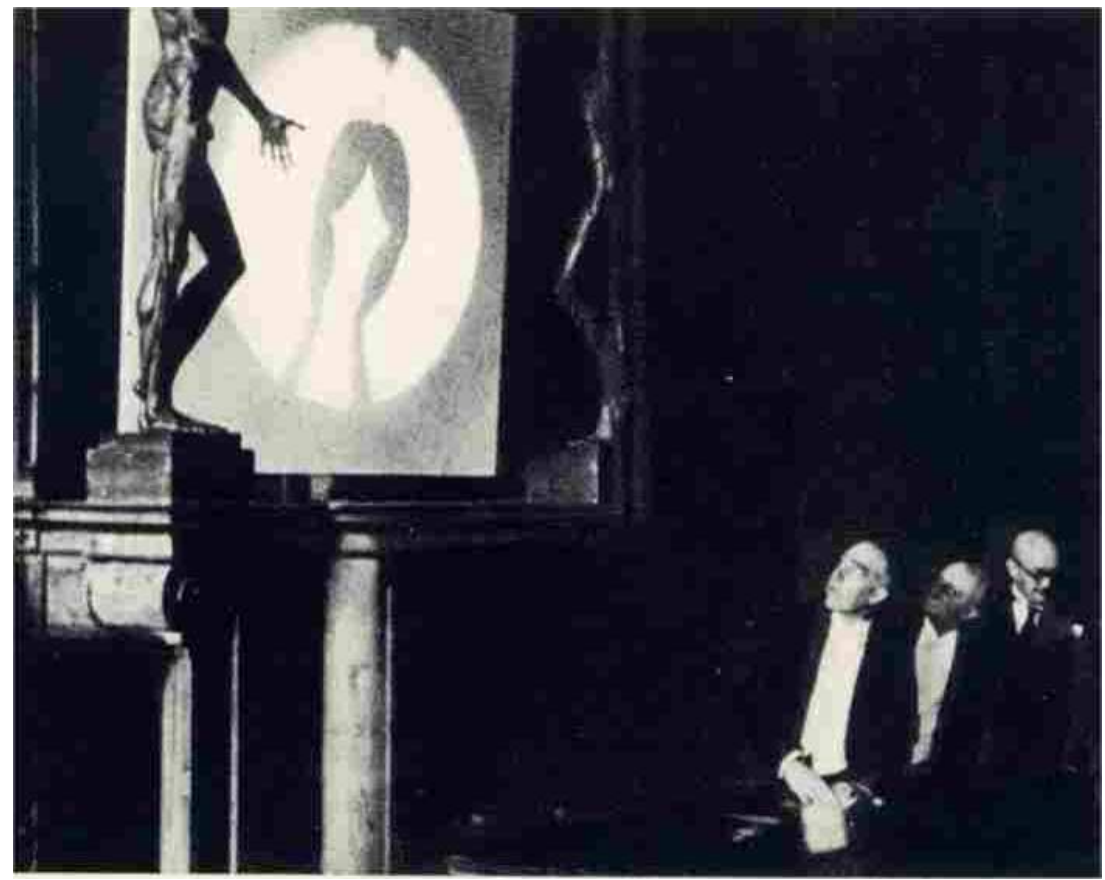

Fig. 8 - Quirino Majorana's replicas of Galvani’s historical experiments on frogs with mono and bimetallic arcs in the anatomical Theater of Archiginnasio Palace in Bologna, where Galvani lectured. Please note Niels Bohr's interested and astonished expression, in front of the didactic-experimental ability shown by Q. Majorana. (Courtesy by “Sapere”, October 31, 1937, p. 265)

Ettore Majorana will comment therefore the event: "I have received also 'Sapere"16. A lot admired, and from many, your speech." (to Quirino November 16, 1937). Among the most relevant aspects in this regard is the extraordinary sensitivity shown by Ettore Majorana (and which may have stemmed from his reading of Galvani's work) toward the role exercised in science by the Principle of Wishful Thinking (when thought is influenced by desire and hence inevitably lacks objectiveness and individual judgment becomes self-deceptive). Galvani himself had been perfectly cognizant of this: "it is easy to be tricked in making experiments and to believe to have seen and to have found what we wish to see and to find." ${ }^{17}$ But, alas, despite his wariness, he was unable to avoid its inexorable effects and he, too, saw what wished to see and discover. In this case as well, Ettore aligned himself with the best scientific traditions of the past. Both Galileo and Newton had been perfectly aware of the traps inherent in our very process of thinking and which lead us, almost inevitably, "to see what is wanted to be seen and to be found". It is an epistemological principle of tantamount importance, which Ettore shows to be well aware of, practically elevating it to the role of a modern "Occam's Razor". Almost such as to be applicable to scientists in general and their cultural sensitivity, based on their effective comprehension and adhesion to this subtle principle, and hence on the basis of their awareness with respect to the validity and applicability of this principle. Failure to recognise this principle strongly limits, though it does not exclude, the possibility of interpreting reality in an adequate fashion. Naturally, even being aware of it is no guarantee, it does not make one immune to

6 "Sapere" (October 31, 1937)

L. Galvani, Opere Scelte, ed. G. Barbensi, UTET Torino 1967, 262 
falling prey to wishful thinking. Just as the cases of Aristotle, Galileo, Galvani, Righi and many others teach us.

\subsection{A conclusive remark}

We would like to conclude our considerations with two quotations we judge to be the most adequate and pertinent with respect to the figure of Ettore Majorana in the history of science and his disappearance. They are both from Enrico Fermi, the greatest personage with whom Ettore consciously exchanged views, deciding, perhaps, to follow other paths than those of his friend and mentor.

The first quotation is due to Giuseppe Cocconi, at the time a young assistant of Fermi, who remembers the opinion of his master about Ettore Majorana: "Because, you know, in the world there are several categories of scientists; people of second and third rank, who make their best but do not go very far ahead. There are also people of first rank, who reach discoveries of great level, of fundamental importance for the development of the science (and here I have clear the impression that in this category he wanted to include himself). But then there are the geniuses, like Galileo and Newton. Well, Ettore was one of those. Majorana had what no other in the world owns; unfortunately he lacked the thing that is instead easy to find in the other men, the simple good sense". ${ }^{18}$

The second is due to Antonio Carrelli, the director of Naples Institute of Physics wherewith Ettore collaborated at that time, who, speaking with Fermi some months after Ettore's disappearance, asked the following question: "Yes, he'd be dead, by now it is passed some time has already gone since we last heard about him...How come is the body of Majorana not found?". Fermi answered Carrelli this way: "But you think that Majorana, with his genius, if he had the problem to disappear and to make his corpse to disappear, would not have been able to resolve it?"19

They seem so remote from each other in both meaning and motivation. In reality, in our opinion, these quotes both express the same admiring wonder of another of the greatest Italian physicists of that time: Enrico Fermi.

\section{Acknowledgements}

I would like to express my best thanks for their help and suggestions to prof. Silvio Bergia, Magda Giorgi, Martina Lodi and Luca Verzelloni. Special thanks also to prof. Carlo Bernardini actual Director of "Sapere". 


\section{References}

[1] Q. Majorana, Su di un fenomeno fotoelettrico constatabile con gli audion, "Rendiconti Accademia dei Lincei" 7 (1928) 801-806

[2] Q. Majorana, Azione della luce su sottili lamine metalliche, "La Ricerca Scientifica", National Research Council, 1 (1935)

[3] Q. Majorana, Agli albori dell'eletricità. Galvani e la scienza moderna, "Sapere”, October 31, 1937, 261-265

[4] Q. Majorana, Ulteriori ricerche sull'azione della luce su sottili lamine metalliche, in "Il Nuovo Cimento", 15 (1938) 573-593

[5] E. Majorana, Lezioni all’Università di Napoli, Bibliopolis, Napoli 1987

[6] L. Galvani, Opere Scelte, ed. G. Barbensi, UTET Torino 1967

[7] L. Castellani, Dossier Majorana, F.lli Fabbri editori, Milano 1974

[8] E. Recami, Il Caso Majorana, Arnoldo Mondatori, Milan 1987 